\title{
The Effect of Balance Training on Cervical Sensorimotor Function and Neck Pain
}

\author{
Konstantin Beinert ${ }^{1,2}$, Wolfgang Taube ${ }^{1}$ \\ ${ }^{1}$ University of Fribourg, Department of Medicine, Movement and Sports Science, Switzerland. ${ }^{2}$ Institute for Applied Science of \\ the Human Movement System, Neustadt a.d. Weinstraße, Germany.
}

\begin{abstract}
The authors' aim was to evaluate the effect of balance training on cervical joint position sense in people with subclinical neck pain. Thirty-four participants were randomly assigned to balance training or to stay active. Sensorimotor function was determined before and after 5 weeks of training by assessing the ability to reproduce the neutral head position and a predefined rotated head position. After balance training, the intervention group showed improved joint repositioning accuracy and decreased pain whereas no effects were observed in the control group. A weak correlation was identified between reduced neck pain intensity and improved joint repositioning. The present data demonstrate that balance training can effectively improve cervical sensorimotor function and decrease neck pain intensity.
\end{abstract}

Keywords: balance exercise, cervical spine, postural control, proprioception

1 $\mathrm{t}$ is well established that the control of upright posture and the control of head and eye movements rely on afferent information arising from different sensory sources such as the vestibular, visual, and proprioceptive system (Horak, 2006). In people with neck pain, coordination of head movements, intersegmental coordination of the vertebrae of the cervical spine, and postural balance were shown to be impaired (Childs et al., 2008). The sensorimotor dysfunction resulted for instance in an enhanced postural sway when measured during unperturbed upright stance (Field, Treleaven, \& Jull, 2008; Stapley, Beretta, Dalla Toffola, \& Schieppati, 2006). Further, a reduced ability to reposition the head in a certain predefined position (Armstrong, McNair, \& Taylor, 2008; Revel, Andre-Deshays, \& Minguet, 1991; Treleaven, Jull, \& Lowchoy, 2005) and an impaired oculomotor control (Tjell \& Rosenhall, 1998) were demonstrated in patients with neck pain. These observations indicate that the proprioceptive information of the neck muscles is crucial to ensure an appropriate control of posture and gaze. In line with this assumption, neck coordination exercises have been shown to improve performance in sensorimotor tasks, alleviate neck pain, and reduce postural sway (Roijezon, Bjorklund, Gergenheim, \& Djupsjobacka, 2008; Jull, Falla, Treleaven, Hodges, \& Vicenzino, 2007; Revel, Minguet, Gregoy, Vaillant, \& Manuel, 1994; Taimela, Takala, Asklof, Seppala, \& Parviainen, 2000). Thus, sensorimotor function of the cervical spine seems to greatly influence posture in general. However, it is not known so far whether this is also true the other way round (i.e., whether balance abilities influence the sensorimotor function of the cervical spine). This is of particular interest as all previous interventions with the aim to improve sensorimotor function of the cervical spine involved consciously performed relocation tasks of the head (Jull et al., 2007; Revel et al., 1994; Roijezon, Bjorklund, Gergenheim, \& Djupsjobacka, 2008; Taimela et al., 2000). In contrast, neck muscles during balance exercises are unconsciously activated while the primary intention is to maintain body equilibrium. Therefore, in the present study we aimed to clarify the influence of five weeks of balance training on the sensorimotor function of the cervical spine. Based on the strong interrelation of neck muscles and posture, it was hypothesized that improved balance performance would reduce the errors when repositioning the head. Furthermore, it was hypothesized that improvements in sensorimotor function would be accompanied by a reduction of the neck pain intensity.

\section{Methods}

\section{Study Participants}

Participants were 34 individuals ( $M$ age $=23 \pm 3$ years $)$ with a history of subclinical neck pain $(M$ duration $=27 \pm$ 14 months). Participants with subclinical neck pain present average pain intensities, but do not display limitations on activity and participation (Lee, Wang, Yao, \& Wang, 2008). Therefore, they are often considered in no need for medical help or advice. Participants were included in the present study, if they had an impaired joint position sense. Joint position sense was considered to be impaired, if the deviation of the head was greater than $3^{\circ}$ in at least 2 of 8 repositioning tasks (Revel et al., 1991). Participants were excluded, if they had any neurological disorders, lower limb injuries, problems with vision and hearing, vestibular pathology, neurological deficits, or diabetes type II (Field et al., 2008; Jull et al., 2007; Treleaven, Jull, \& LowChoy, 2006). Participants provided written informed consent before participating in the experiment. The study was approved by the ethic committee of the University of Freiburg and was conducted in accordance with the declaration of Helsinki.

\section{Joint Position Sense}

Joint position sense (JPS) was tested with a target matching task, which was first described by Revel et al. (1991). A cervical goniometry (CMS, Hamburg, Germany) with a laser pointer (NOBO; ACCO, Schorndorf, Germany) was placed on the head of the participants (Figure 1).

Correspondence address: Konstantin Beinert, University of Fribourg, Department of Medicine, Movement and Sport Science, Switzerland.e-mail: konstantin.beinert@unifr.ch 


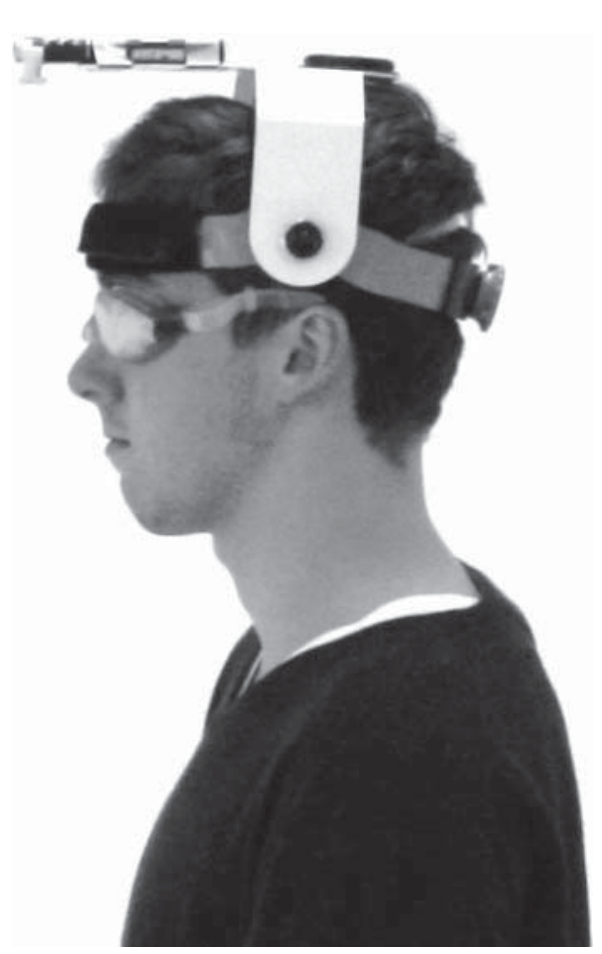

FIGURE 1. Experimental apparatus for measurement of the NHP (neutral head position) and RHP (rotated head position). The participant is seated $90 \mathrm{~cm}$ in front of the target, vision occluded. The laser pointer is fixed on an a cervical goniometer.

Participants were seated in an unsupported straight position on a chair with a distance of $90 \mathrm{~cm}$ to the target (Revel et al., 1991). The target was an adjustable board of $1.5 \times 1 \mathrm{~m}$, which was placed so that the center of the board was at eye level for each individual participant.

The position when participants looked straight ahead was defined as the neutral head position (NHP). For testing the cervical joint position sense, participants were instructed to move their head with open eyes from the NHP into four predefined positions before relocating the head into the NHP with closed eyes. The defined positions were (a) rotation to the right, (b) rotation to the left, (c) extension, and (d) flexion.

The second test to assess joint position sense of the cervical spine resembled the first test but participants started from a prerotated head position and had to come back to this position. The pre-rotated head positions (RHP) were (a) rotation of $45^{\circ}$ to the right, (b) rotation of $45^{\circ}$ to the left, (c) rotation of $45^{\circ}$ in extension, and (d) rotation of $45^{\circ}$ in flexion. From these positions participants were instructed to move their head into the NHP with eyes open before relocating the head with closed eyes into the initial RHP. For each task, two repetitions were performed as warming up and the third repetition was measured. The participants indicated verbally when they thought that they had reached their target position. The location of the laser point at that instant was marked and the deviation to the actual target position was determined in centimeters and degrees. Thus, for each target position (NHP and RHP) four trials were recorded and averaged for each participant.

Recently it was shown that this measurement technique not only demonstrated high correlation with ultrasound-based measurements $(r=.95)$ but also had the same discriminative ability to differentiate healthy control form neck pain participants (Roren et al., 2009). Test-retest reliability was demonstrated to be good to excellent with an interclass correlation ranging from 0.52 to 0.81 (Pinsault et al., 2008). After initial testing, participants were randomly allocated into an intervention group and a control group. While the participants of the intervention group participated in a balance training program, members of the control group maintained their usual physical activity level. The researcher executing the measurements was blinded to participant group for outcome assessments and statistical analyses.

\section{Neck Pain Assessment}

Neck pain intensity was assessed using a numeric rating scale (NRS), which is a reliable and valid measurement tool, especially in small sample sizes (Jensen, Turner, Romano, \& Fisher, 1999). Participants had to indicate their level of neck pain on a $10 \mathrm{~cm}$ NRS anchored with 0 (no pain) and 10 (the worst possible pain imaginable).

\section{Intervention}

The intervention group trained over a period of five weeks, three times per week, with a total of 15 training sessions. Each session lasted for $15 \mathrm{~min}$ and was documented, surveyed and supervised by the authors of the study. The intervention consisted of three balance tasks: single leg stance, tandem stance and standing on a wobble board (Figure 2). Each task was performed $20 \mathrm{~s}$ with a 10 -s break in between. It was taken care that the body position was comparable in all trials and was similar among participants. Participants were instructed to hold their head upright, align their arms to their body and slightly bent their knee. Both legs were alternately trained. When participants were able to maintain balance for $20 \mathrm{~s}$, the difficulty of the task was successively increased. First, participants had to perform eye movements in the sagittal and transverse plane without moving their head. In the next step, eyes had to be closed. Finally, the stability of the support surface was further decreased by reducing the base of support while participants stood with closed eyes. The control group maintained their normal physical activity throughout the experimental period.

\section{Data Analysis and Statistics}

To identify potential differences between groups in the pre-measurement, one-way analyses of variance (ANOVAs) were conducted for each parameter (head repositioning, pain). The effect of balance training on the ability to reproduce predefined head positions was tested by a repeated measures ANOVA procedure with the within-participant 

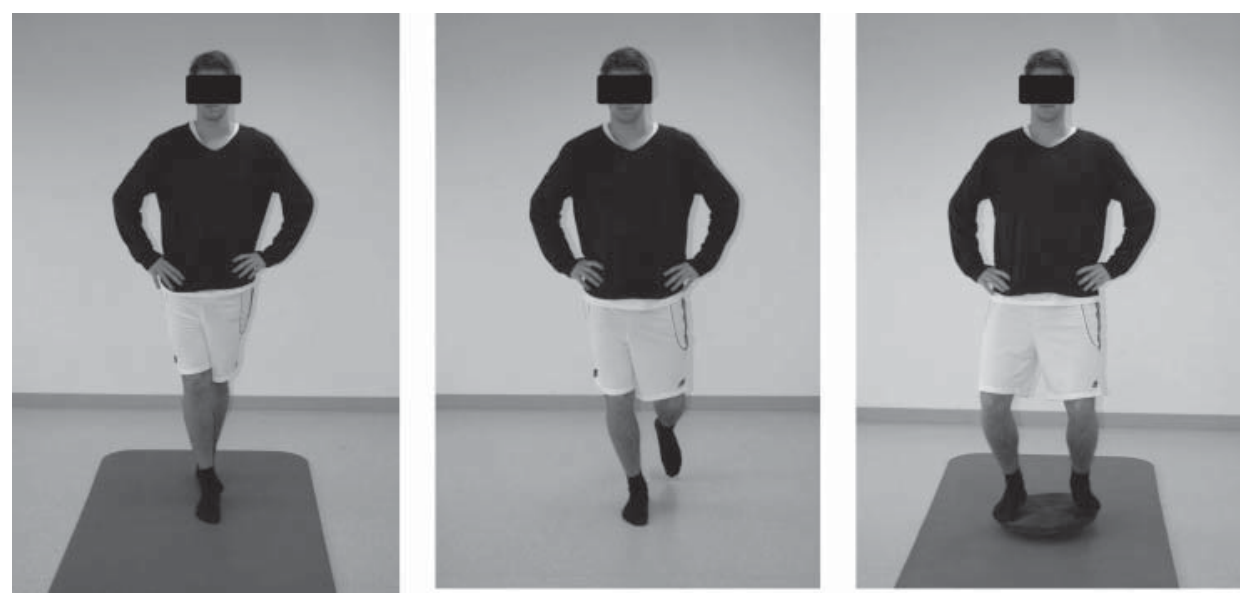

FIGURE 2. Three balance tasks were performed: single leg stance, tandem stance, and standing on a wobble board.

factors of time (pre- vs. posttraining measurement) and condition (NHP vs. RHP) and the between-participant factor of group (INT vs. CON): 2 Time $\times 2$ Condition $\times 2$ Group. In case of significant $F$ values, Bonferroni-corrected post hoc tests were performed to identify changes for each condition (NHP vs. RHP). Correlations between changes in head repositioning performance and changes in neck pain perception were assessed by means of the Pearson correlation coefficient. To identify differences in pain intensity in pre- and post-measurements, Bonferroni-corrected Student $t$ tests were conducted.

All variables were expressed as mean values \pm standard deviation, if not indicated differently. The significance level was set to .05 for all tests. SPSS software version 16.0 was used for all statistical analysis.

\section{Results}

All 34 participants who were recruited for this study completed pre- and posttest measurement. The exercise program enhanced JPS acuity and reduced pain intensity.

\section{Joint Position Sense}

Before training, there was no statistical difference between intervention and control group regarding the JPS (for NHP and RHP $p>.1$ ). The acuity of repositioning the head in a predefined position (either NHP or RHP) was group specifically altered after training indicated by a significant time by group interaction (time pre/post by group CON/INT $_{\text {) }} F(1,33)=$ $5.65 ; p=.023$. For the intervention group, post -hoc analysis revealed significantly decreased deviations in repositioning for the RHP by improving accuracy from $3.04^{\circ} \pm 0.73^{\circ}$ to $1.88^{\circ} \pm 0.57^{\circ}(p=.005$; Figure $3 \mathrm{~A})$. In the NHP task, participants improved accuracy from $3.37^{\circ} \pm 1.6^{\circ}$ to $2.43^{\circ} \pm$ $0.8^{\circ}(p=.026$; Figure $3 \mathrm{~B})$. No significant changes over time could be seen in the control group (always $p>.1$ ). Thus, the acuity to reposition the head improved exclusively in the intervention group indicating a beneficial effect of balance training on this parameter.

\section{Neck Pain}

Neck pain intensity ranged from 2 to $10(M=4.66 \pm 2.35)$. Before training, neck pain intensity on the NRS ranged from 2 to $8(M=3.65 \pm 1.90)$ in the intervention group and 2 to $10(M=5.61 \pm 2.44)$ in the control group and was different between groups $(p=.013)$. This difference was due to the random allocation of our participants into training and control group.

After balance training, neck pain intensity decreased significantly in the intervention group and ranged from 0.5 to 4 on the NRS (pre $3.65 \pm 1.90$ vs. post $1.97 \pm 0.91 ; p=.011$; Figure 4). Neck pain persisted in the control group ranging from 1 to 9 (pre $5.61 \pm 2.44$ vs. post $4.78 \pm 2.71 ; p=.89$; Figure 3).

\section{Correlation Between Pain Intensity and Joint Position Sense}

After balance training, there was a weak but significant positive correlation between reduction in neck pain and reduction in the repositioning error in the RHP task $(r=.43$, $p=.042$; Figure 5). No correlation was found between changes in neck pain intensity and changes in the repositioning error when measured in the NHP task $(r=-.2$, $p=.4)$.

\section{Discussion}

The primary aim of the present study was to investigate the influence of balance training on cervical sensorimotor 
A

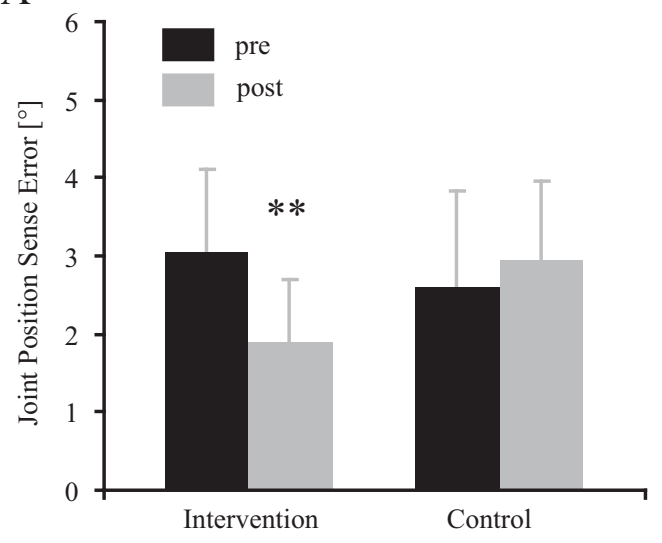

Rotated Head Position
$\mathrm{B}$

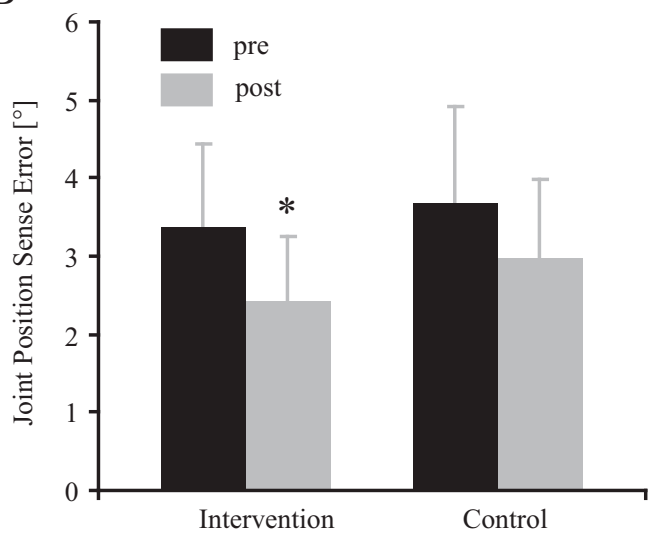

Neutral Head Position

FIGURE 3. Mean values for improved joint position sense acuity for the RHP task (A) and the NHP task (B) of the intervention and control group. Black bars represent preintervention values (Pre). Gray bars represent post intervention values (Post). Significant differences between pre- and postintervention values are marked with an asterisk $\left({ }^{*} p<.05 ;{ }^{* *} p<.01\right)$.

function. The present data indicate that balance exercises were indeed efficient to improve joint repositioning acuity. The secondary outcome measure aimed to evaluate changes in neck pain intensity in response to balance training. Neck pain intensity was significantly reduced in the training group but remained unaltered in the control group. The results of the present study demonstrate that improvements in joint position sense of the cervical spine and reduction of neck pain do not necessarily have to be associated to head relocation exercises (Humphreys \& Irgens, 2002; Jull et al., 2007) but

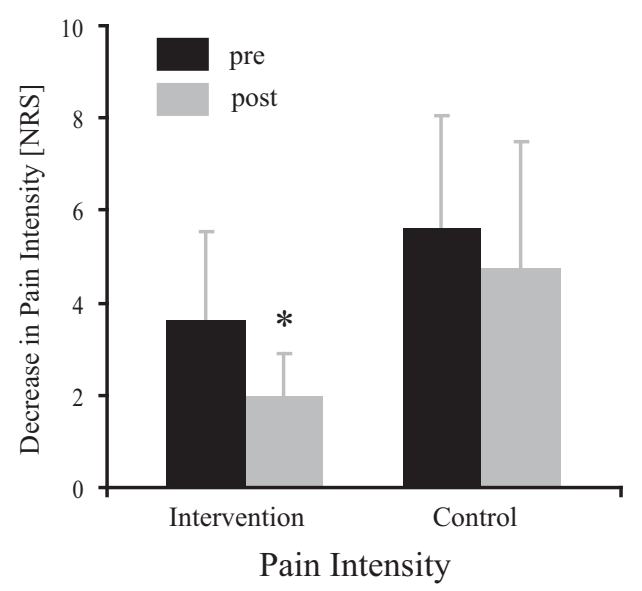

FIGURE 4. Mean values for pain intensity of the intervention and control group. Black bars represent preintervention values (Pre) whereas gray bars represent postintervention values (Post). Significant differences between pre and post intervention values are marked with an asterisk $\left({ }^{*} \alpha<.01\right)$. can also be obtained by balance tasks. In contrast to head relocation exercises, in which the primary goal is to move the head in a specific and predefined way, balance exercises force the participants to focus on maintaining or regaining equilibrium in space. Thus, it may be speculated that little attention is consciously allocated to the cervical spine during balancing.

\section{Balance Training and Joint Position Sense}

It is well known that influencing the somatosensory system of the cervical spine influences balance performance. For instance, it was demonstrated that balance performance can be deteriorated by fatiguing or vibrating the neck muscles (Gosselin, Rassoulian, \& Brown, 2004; Kavounoudias, Gilhodes, Roll, \& Roll, 1999; Schieppati, Nardone, \& Schmid, 2003). Conversely, improved balance performance was reported after neck coordination exercises (Roijezon et al., 2008). Thus, there has been good evidence for a close relationship between the somatosensory system of the cervical spine and postural control. However, so far, all previous interventions were specifically targeting the neck muscles and postural control was assessed as the outcome measure. In contrast, the present data highlight for the first time that training of balance skills may also beneficially alter sensorimotor function of neck muscles indicated by improved cervical joint position sense acuity. Independent of the measurement, thus, independent whether repositioning errors were determined in the NHP or the RHP task, participants of the balance training group decreased their error rate whereas the control group showed no adaptation.

In summary, the effects of balance training on cervical JPS seem to be comparable to those reported after 


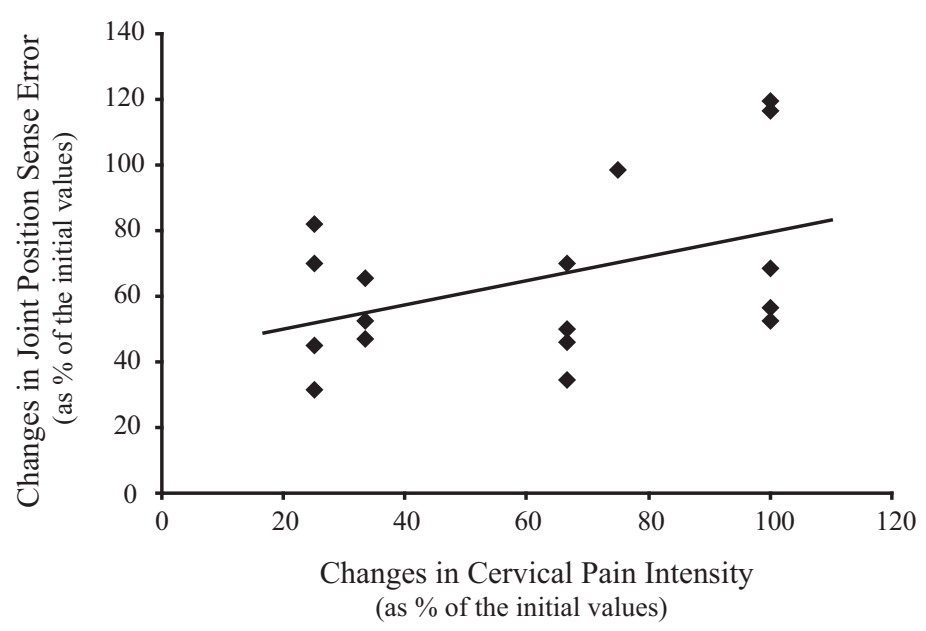

FIGURE 5. Positive significant correlation between reduction in neck pain and reduction in joint position sense error.

interventions incorporating relocation tasks of the cervical spine and the head (Humphreys \& Irgens, 2002; Jull et al., 2007). In the study of Jull et al. (2007), participants had to consciously control their neck muscle activity while producing predefined force levels in a craniocervical flexion task. For this purpose, participants received feedback about their neck muscle activity by their therapist (Jull, Falla, Treleaven, Sterling, \& O'Leary, 2004). Participants in the study of Humphreys et al. (2002) performed conscious gaze stabilization tasks while performing head rotations in the sagittal plane. Thus, all previous studies had in common that participants had to consciously relocate their head into specific target positions. In contrast, it may be speculated that participants directed very little if no attention to the specific position of their head in space during balancing. Thus, balance exercises in the present study probably involved much more involuntary activation of neck muscles as the primary intention was to keep the body's center of mass over the base of support. It may therefore be speculated that not only the intention during task execution was different in the present study compared to previous interventions targeting the cervical spine but also the neural control and thus, the muscular activation. In contrast to cervical relocation tasks, balance training involves whole body postural reactions, which are not performed in a standardized way. Thus, muscle activation during postural reactions is probably not only mainly unconsciously performed but also in a highly variable way. Both factors may contribute to differential neck muscle activities during postural tasks compared to consciously performed exercises with the aim to relocate the head to predefined targets. However, it has to be mentioned that no study has compared activities of neck muscles in relocation and balance tasks, so far. Thus, further research is needed to evaluate the effectiveness of balance exercises to distract attention from the impaired body part (cervical spine). This might be beneficial for participants with high levels of fear of neck movements.

\section{Comparison of Sensorimotor Function in NHP and RHP Tasks}

Previous studies have highlighted that targeted interventions are able to improve the sensorimotor function of the cervical spine when the NHP task was used as the outcome measure. The present study did not only test repositioning abilities in the NHP task, but also in the RHP task. Participants of the balance training group improved both qualities, with stronger effects in the RHP task compared to the NHP task. Thus, it may be speculated that the two different repositioning tasks may display different perceptive abilities of the somatosensory system. In everyday live, the neutral head position is considered to be the normal head position and therefore, the NHP-task may display the congruence of afferent input from the left and right side of the body in relation to a long time memory of the midpoint (neutral position) of the head. Failure in reproducing the NHP by means of a constant pattern of undershoot or overshoot may reflect a disrupted cortical representation of NHP. In contrast, the RHP-task may test the ability to memorize a specific head position for only a short time as rotated head positions are usually not maintained over a longer period of time. This assumption seems reasonable due to findings in patients with chronic low back pain. In those patients, altered cortical representation of their lower back has been found, indicated by a shift of this representation in the somatosensory homunculus (Flor, Braun, Elbert, \& Birbaumer, 1997). Furthermore, differences in the primary motor cortex have been observed between healthy participants and lower back pain patients with impaired sensorimotor control (for a review, see Wand 
et al., 2011). Patients with impaired sensorimotor function and occurrence of pain displayed expanded and shifted areas of motor cortical activity when activating the corresponding muscle(s) (transversus abdominis; Tsao, Galea, \& Hodges, 2010). Based on those lower back pain studies it may be assumed that even patients with mild neck pain may display changes within the cortical representation, which in turn may influence especially their perception of the midpoint and, thus, their performance in the NHP task.

\section{Balance Training and Neck Pain}

While it is established, that neck pain can impair balance performance in people with traumatic (Sjostrom et al., 2003; Stapley et al., 2006; Treleaven et al., 2005) and idiopathic neck pain (Field et al., 2008), the influence of balance interventions on neck pain intensity has not been demonstrated before. So far, the effects of balance training have been seen in a reduction in the recurrence of ankle sprains in chronic ankle instability (Hale, Hertel, \& Olmsted-Kramer, 2007; Sefton, Yarar, Hicks-Little, Berry, \& Cordova, 2011), a reduction in giving way episodes in ACL injured people (Fitzgerald, Axe, \& Snyder-Mackler, 2000), a reduction of risk of falling in elderly people (Granacher et al., 2010), and a reduced incidence of knee and ankle injuries (Zech et al., 2010). Furthermore, balance trained participants displayed an improved postural control, an enhanced rate of force development and an improved jump performance (Gruber et al., 2007; Taube, Gruber et al., 2007; Taube, Kullmann et al., 2007). Thus, although balance training has previously been shown to improve sensorimotor function of the lower extremity in a broad way (for review Taube, Gruber, \& Gollhofer, 2008), the present study displays an absolutely new aspect of this kind of training: It seems that balance training positively influences sensorimotor function of the cervical spine and neck pain intensity — at least in young participants with subclinical neck pain. As we observed a weak but nevertheless significant correlation between decreases in neck pain and improvements in sensorimotor function it may be speculated that both phenomena share some common features.

\section{Mechanisms That May Underlay the Interrelation of Sensorimotor Function and Pain}

The question remains, how sensorimotor function and pain perception are potentially interrelated. When considering sensorimotor circuits, neural activity of supraspinal centers after stimulation of foot proprioceptors (i.e., muscle spindles) was demonstrated to correlate with balance performance (Goble et al., 2011). In particular, greater balance performance was associated with greater activity in parietal, frontal, and insular cortical areas, as well as structures within the basal ganglia. Similarly, training studies indicated that cortical structures (e.g., motor cortex, supplementary motor area) adapt in response to balance exercises (Taube et al., 2008; Taubert et al., 2010). It might therefore be proposed that processing in those structures is important to ensure balance control. Interestingly, some of these brain areas are also known to be involved in chronic pain processing (Apkarian, Baliki, \& Geha, 2009; Treede, Apkarian, Bromm, Greenspan, \& Lenz, 2000). Thus, some supraspinal somatosensory representations seem to be closely related to both sensorimotor function and pain perception. As pain can influence both the corresponding somatosensory cortical representation of the painful body area (Flor et al., 1997) and the sensorimotor information processing (Flor, 2012), it may be speculated that balance training affected supraspinal structures responsible for both sensorimotor control and pain perception.

\section{Limitations}

Although the researcher executing the measurements was blinded to participant group for outcome assessments and statistical analyses, the present results should not be generalized. As the present study was done with a relatively small number of participants $(n=34)$ who displayed only subclinical levels of neck pain, further studies have to indicate whether patients with more serious impairments of the cervical spine can also benefit from balance training interventions. Furthermore, the present measurements only allow assumptions about the underlying neural mechanisms but cannot provide insights about training-related plasticity.

\section{Conclusion}

In contrast to cervical repositioning exercises, which are most often performed with a special helmet and a predefined goal, balance training is easy to apply and can be performed everywhere without complex equipment. Furthermore, the accomplishment of postural tasks demands whole body movements and distracts people with neck pain away from their impairment. Thus, the intention of the sensorimotor system is differently challenged during balance tasks than in consciously performed cervical relocation tasks. Therefore, it may be speculated that neck muscles are differently recruited during whole body postural tasks and that the improved cervical joint position sense and reduction in cervical pain intensity may therefore differ in the present study from the mechanisms proposed in previous studies evaluating the effect of conscious relocation tasks on the cervical spine. Nevertheless, the muscular activation induced by balance training seems to be efficient to counteract impaired joint position sense and neck pain of the cervical spine.

\section{REFERENCES}

Apkarian, A. V., Baliki, M. N., \& Geha, P. Y. (2009). Toward a theory of chronic pain. Progress in Neurobiology, 87, 81-97. doi:S03010082(08)00113-5 [pii]10.1016/j.pneurobio.2008.09.018

Armstrong, B., McNair, P., \& Taylor, D. (2008). Head and neck position sense. Sports Medicine, 38, 101-117. doi:3822 [pii]

Childs, J. D., Cleland, J. A., Elliott, J. M., Teyhen, D. S., Wainner, R. S., Whitman, J. M., ... Flynn, T. W. (2008). Neck pain: Clinical 
practice guidelines linked to the International Classification of Functioning, Disability, and Health from the Orthopedic Section of the American Physical Therapy Association. Journal of Orthhopedic and Sports Physical Therapy, 38, A1-A34. doi:1454 [pii]

Field, S., Treleaven, J., \& Jull, G. (2008). Standing balance: A comparison between idiopathic and whiplash-induced neck pain. Manual Therapy, 13, 183-191. doi:S1356-689X(07)000021 [pii]10.1016/j.math.2006.12.005

Fitzgerald, G. K., Axe, M. J., \& Snyder-Mackler, L. (2000). The efficacy of perturbation training in nonoperative anterior cruciate ligament rehabilitation programs for physical active individuals. Physical Therapy, 80, 128-140.

Flor, H. (2012). New developments in the understanding and management of persistent pain. Current Opinions in Psychiatry, 25, 109-113. doi:10.1097/YCO.0b013e3283503510

Flor, H., Braun, C., Elbert, T., \& Birbaumer, N. (1997). Extensive reorganization of primary somatosensory cortex in chronic back pain patients. Neuroscience Letters, 224, 5-8. doi:S03043940(97)13441-3 [pii]

Goble, D. J., Coxon, J. P., Van Impe, A., Geurts, M., Doumas, M., Wenderoth, N., \& Swinnen, S. P. (2011). Brain activity during ankle proprioceptive stimulation predicts balance performance in young and older adults. Journal of Neuroscience, 31, 16344-16352. doi:31/45/16344 [pii]10.1523/JNEUROSCI. 4159-11.2011

Gosselin, G., Rassoulian, H., \& Brown, I. (2004). Effects of neck extensor muscles fatigue on balance. Clinical Biomechanics (Bristol, Avon), 19, 473-479. doi:10.1016/j.clinbiomech. 2004.02.001S0268003304000312 [pii]

Granacher, U., Muehlbauer, T., Bridenbaugh, S., Bleiker, E., Wehrle, A., \& Kressig, R. W. (2010). Balance training and multitask performance in seniors. International Journal of Sports Medicine, 31, 353-358. doi:10.1055/s-0030-1248322

Gruber, M., Gruber, S. B., Taube, W., Schubert, M., Beck, S. C., \& Gollhofer, A. (2007). Differential effects of ballistic versus sensorimotor training on rate of force development and neural activation in humans. Journal of Strength Conditioning Research, 21, 274-282. doi:R-20085 [pii]10.1519/R-20085.1

Hale, S. A., Hertel, J., \& Olmsted-Kramer, L. C. (2007). The effect of a 4-week comprehensive rehabilitation program on postural control and lower extremity function in individuals with chronic ankle instability. Journal of Orthopedic and Sports Physical Therapy, 37, 303-311.

Horak, F. B. (2006). Postural orientation and equilibrium: What do we need to know about neural control of balance to prevent falls? Age and Ageing, 35, ii7-ii11. doi:35/suppl_2/ii7 [pii]10.1093/ageing/afl077

Humphreys, B. K., \& Irgens, P. M. (2002). The effect of a rehabilitation exercise program on head repositioning accuracy and reproted levels of pain in chronic neck pain subjects. Journal of Whiplash \& Related Disorders, 1(1).

Jensen, M. P., Turner, J. A., Romano, J. M., \& Fisher, L. D. (1999). Comparative reliability and validity of chronic pain intensity measures. Pain, 83, 157-162. doi:S0304395999001013 [pii]

Jull, G., Falla, D., Treleaven, J., Hodges, P., \& Vicenzino, B. (2007). Retraining cervical joint position sense: The effect of two exercise regimes. Journal of Orthopedic Research, 25, 404-412. doi:10.1002/jor.20220

Jull, G., Falla, D., Treleaven, J., Sterling, M. M., and O'Leary, S. P. (2004). A therapeutic exercise approach for cervical disorders. In J. G. Boyling \& G. Jull (Ed.), Grieve's modern manual therapy: The vertebral column (pp. 451-470). Edinburgh, Scotland: Churchill Livingstone.

Kavounoudias, A., Gilhodes, J. C., Roll, R., \& Roll, J. P. (1999). From balance regulation to body orientation: Two goals for mus- cle proprioceptive information processing? Exp Brain Research, $124,80-88$.

Lee, H. Y., Wang, J. D., Yao, G., \& Wang, S. F. (2008). Association between cervicocephalic kinesthetic sensibility and frequency of subclinical neck pain. Manual Therapy, 13, 419-425. doi:S1356689X(07)00080-X [pii]10.1016/j.math.2007.04.001

Pinsault, N., Fleury, A., Virone, G., Bouvier, B., Vaillant, J., \& Vuillerme, N. (2008). Test-retest reliability of cervicocephalic relocation test to neutral head position. Physiotherapy Theory and Practice, 24, 380-391. doi:903021724 [pii]10.1080/ 09593980701884824

Revel, M., Andre-Deshays, C., \& Minguet, M. (1991). Cervicocephalic kinesthetic sensibility in patients with cervical pain. Archives of Physical Medicine and Rehabilitation, 72, 288-291. doi:0003-9993(91)90243-C [pii]

Revel, M., Minguet, M., Gregoy, P., Vaillant, J., \& Manuel, J. L. (1994). Changes in cervicocephalic kinesthesia after a proprioceptive rehabilitation program in patients with neck pain: A randomized controlled study. Archives of Physical Medicine and Rehabilitation, 75, 895-899.

Roijezon, U., Bjorklund, M., Bergenheim, M., \& Djupsjobacka, M. (2008). A novel method for neck coordination exercise-a pilot study on persons with chronic non-specific neck pain. Journal of Neuroengineering and Rehabilitation, 5, 36. doi:1743-0003-5-36 [pii]10.1186/1743-0003-5-36

Roren, A., Mayoux-Benhamou, M. A., Fayad, F., Poiraudeau, S., Lantz, D., \& Revel, M. (2009). Comparison of visual and ultrasound based techniques to measure head repositioning in healthy and neck-pain subjects. Manual Therapy, 14, 270-277. doi:S1356-689X(08)00057-X [pii]10.1016/j.math.2008.03.002

Schieppati, M., Nardone, A., \& Schmid, M. (2003). Neck muscle fatigue affects postural control in man. Neuroscience, 121, 277-285. doi:S0306452203004391 [pii]

Sefton, J. M., Yarar, C., Hicks-Little, C. A., Berry, J. W., \& Cordova, M. L. (2011). Six weeks of balance training improves sensorimotor function in individuals with chronic ankle instability. Journal of Orthopedic and Sports Physical Therapy, 41, 81-89. doi:2513 [pii]10.2519/jospt.2011.3365

Sjostrom, H., Allum, J. H., Carpenter, M. G., Adkin, A. L., Honegger, F., \& Ettlin, T. (2003). Trunk sway measures of postural stability during clinical balance tests in patients with chronic whiplash injury symptoms. Spine (Philadelphia), 28, 1725-1734. doi:10.1097/01.BRS.0000083170.34304.A3

Stapley, P. J., Beretta, M. V., Dalla Toffola, E., \& Schieppati, M. (2006). Neck muscle fatigue and postural control in patients with whiplash injury. Clinical Neurophysiology, 117, 610-622. doi:S1388-2457(05)00472-4 [pii]10.1016/j.clinph.2005.11.007

Taimela, S., Takala, E. P., Asklof, T., Seppala, K., \& Parviainen, S. (2000). Active treatment of chronic neck pain: A prospective randomized intervention. Spine (Philadelphia), 25, 1021-1027.

Taube, W., Gruber, M., Beck, S., Faist, M., Gollhofer, A., \& Schubert, M. (2007). Cortical and spinal adaptations induced by balance training: Correlation between stance stability and corticospinal activation. Acta Physiologica (Oxford), 189, 347-358. doi:APS1665 [pii]10.1111/j.1365-201X.2007.01665.x

Taube, W., Gruber, M., \& Gollhofer, A. (2008). Spinal and supraspinal adaptations associated with balance training and their functional relevance. Acta Physiologica (Oxford), 193, 101-116. doi:APS1850 [pii]10.1111/j.1748-1716.2008.01850.x

Taube, W., Kullmann, N., Leukel, C., Kurz, O., Amtage, F., \& Gollhofer, A. (2007). Differential reflex adaptations following sensorimotor and strength training in young elite athletes. International Journal of Sports Medicine, 28, 999-1005. doi:10.1055/s-2007-964996

Taubert, M., Draganski, B., Anwander, A., Muller, K., Horstmann, A., Villringer, A., \& Ragert, P. (2010). Dynamic properties of human brain structure: Learning-related changes in cortical 
areas and associated fiber connections. Journal of Neuroscience, 30, 11670-11677. doi:30/35/11670 [pii]10.1523/JNEUROSCI. $2567-10.2010$

Tjell, C., \& Rosenhall, U. (1998). Smooth pursuit neck torsion test: A specific test for cervical dizziness. American Journal of Otolaryngology, 19, 76-81.

Treede, R. D., Apkarian, A. V., Bromm, B., Greenspan, J. D., \& Lenz, F. A. (2000). Cortical representation of pain: Functional characterization of nociceptive areas near the lateral sulcus. Pain, 87, 113-119. doi:S0304-3959(00)00350-X [pii]

Treleaven, J., Jull, G., \& Lowchoy, N. (2005). Standing balance in persistent whiplash: A comparison between subjects with and without dizziness. Journal of Rehabilitation Medicine, 37, 224-229. doi:RM248275U6P140JK [pii]10.1080/ 16501970510027989

Treleaven, J., Jull, G., \& Lowchoy, N. (2006). The relationship of cervical joint position error to balance and eye movement disturbances in persistent whiplash. Manual Therapy, 11, 99-106. doi:S1356-689X(05)00042-1 [pii]10.1016/j.math.2005.04.003
Tsao, H., Galea, M. P., \& Hodges, P. W. (2010). Driving plasticity in the motor cortex in recurrent low back pain. European Journal of Pain, 14, 832-839. doi:S1090-3801(10)00019-4 [pii]10.1016/j.ejpain.2010.01.001

Wand, B. M., Parkitny, L., O'Connell, N. E., Luomajoki, H., McAuley, J. H., Thacker, M., \& Moseley, G. L. (2011). Cortical changes in chronic low back pain: Current state of the art and implications for clinical practice. Manual Therapy, 16, 1520. doi:S1356-689X(10)00111-6 [pii]10.1016/j.math.2010.06. 008

Zech, A., Hubscher, M., Vogt, L., Banzer, W., Hansel, F., \& Pfeifer, K. (2010). Balance training for neuromuscular control and performance enhancement: A systematic review. Journal of Athletic Training, 45, 392-403. doi:10.4085/1062-6050-45.4. 392 